\title{
The membrane complement regulatory protein CD59 promotes tumor growth and predicts poor prognosis in breast cancer
}

\author{
QIANWEN OUYANG ${ }^{1-5^{*}}$, LONG ZHANG ${ }^{3,4,6^{*}}$, YIZHOU JIANG ${ }^{2-4 *}$, XIAOJIAN NI ${ }^{2-4 *}$, SHENG CHEN ${ }^{2-4 *}$, \\ FUGUI YE ${ }^{7}$, YIQUN DU ${ }^{4,8}$, LIANG HUANG ${ }^{2-4}$, PEIPEI DING ${ }^{3,4,6}$, NA WANG $^{3}$, \\ CHAOQUN YANG $^{9}$, TIANBAO HUANG ${ }^{10}$, YUJING SUN ${ }^{3}$, SHAN LI $^{2-4}$, YUN XIA ${ }^{1}$, \\ WEIGUO $\mathrm{HU}^{3,4,6}$, RONGCHENG LUO ${ }^{5}$ and ZHIMING SHAO ${ }^{2-4,6}$
}

\author{
${ }^{1}$ Department of Breast Surgery, The Third Hospital of Nanchang, China Jiangxi Province Key Laboratory for Breast Diseases, \\ Nanchang, Jiangxi; ${ }^{2}$ Department of Breast Surgery, Key Laboratory of Breast Cancer in Shanghai, \\ ${ }^{3}$ Fudan University Cancer Institute, Shanghai Cancer Center, ${ }^{4}$ Department of Oncology, Shanghai Medical College, \\ Fudan University, Shanghai; ${ }^{5}$ Cancer Center, Traditional Chinese Medicine-Integrated Hospital, \\ Southern Medical University, Guangzhou, Guangdong; ${ }^{6}$ Institutes of Biomedical Science, Fudan University, \\ Shanghai; ${ }^{7}$ Department of General Surgery, Affiliated Union Hospital of Fujian Medical University, \\ Union Clinical School, Fujian Medical University, Fujian; Departments of ${ }^{8}$ Medical Oncology, \\ Shanghai Cancer Center and ${ }^{9}$ Hand Surgery, Huashan Hospital, Fudan University; ${ }^{10}$ Department of Urology, \\ Shanghai Tenth People's Hospital, Tongji University, Shanghai, P.R. China
}

Received October 22, 2015; Accepted February 1, 2016

DOI: $10.3892 /$ ijo.2016.3408

\begin{abstract}
Breast cancer is the most prevalent type of cancer among women. CD59, a membrane complement regulatory protein, has been demonstrated to be overexpressed in most solid tumors, where it facilitates tumor cell escape from complement surveillance. However, the role of CD59 in breast cancer growth and clinical prognosis is not fully revealed. To investigate the role of CD59 in breast cancer growth and prognostic significance, we knocked down CD59 in a breast cancer cell line that is highly metastatic to the lungs, MDA-MB-231-HM. Cell growth was measured in vitro and in vivo using a xenograft model. In addition, clinical data on a cohort of 120 patients with or without lung metastasis was analyzed based on CD59 expression, which was detected by
\end{abstract}

Correspondence to: Professor Zhiming Shao, Department of Breast Surgery, Key Laboratory of Breast Cancer in Shanghai, Shanghai Cancer Center, Fudan University, 270 Dong-An Road, Shanghai 200032, P.R. China

E-mail: zhi_ming_shao@163.com

Professor Rongcheng Luo, Cancer Center, Traditional Chinese Medicine-Integrated Hospital, Southern Medical University, 1838 Guangzhou N Avenue, Baiyun, Guangzhou, Guangdong 510515, P.R. China

E-mail: luorc01@163.com

*Contributed equally

Key words: MDA-MB-231-HM, CD59, tumor growth, breast cancer, poor prognosis immunohistochemistry. Knockdown of CD59 significantly inhibited MDA-MB-231-HM cell growth both in vitro and in vivo. An analysis of clinical data on 120 patients revealed that patients with CD59 overexpression may have a worse prognosis. CD59 may therefore be a prognostic biomarker for poor outcome in breast cancer patients.

\section{Introduction}

Breast cancer is the most prevalent cancer among women, with an estimated 232,670 new cases diagnosed this year (29\% of all malignancies in women); breast cancer ranks second overall in mortality (15\%), with an estimated 40,000 deaths, behind lung and bronchus carcinoma (1). Diagnosis at an early stage is beneficial for patients $(2,3)$. Although there are prognostic factors for long-term survival $(4,5)$, breast cancer metastasis, especially to the lungs, remains a significant clinical challenge; the median survival of these patients is $<2$ years after diagnosis $(6,7)$. Therefore, there is an unmet need for prognostic biomarkers that can predict breast cancer prognosis.

Metastasis of tumor cells involves the physical translocation of cancer cells to a distant organ and the survival of metastatic cancer cells at a distant site (8); during the invasion process, cancer cells attempt to escape immune surveillance. The complement system is the pillar of innate immunity $(9,10)$; the activation of complement leads to the efficient clearance of 'non-self' and unwanted 'self' antigens, including those on tumor cells (11), via versatile mechanisms, such as membrane attack complex (MAC)-mediated cytolysis (12). To escape complement attack, tumor cells express high levels of membrane complement regulatory proteins (mCRPs) (13). $\mathrm{CD} 59$, the sole membrane regulator in the terminal stage of 
complement activation, is considered to be the most effective $\mathrm{mCRP}$ at protecting cancer cells by inhibiting MAC formation (14). CD59 is widely expressed, and its expression levels are higher in almost all cancer cells than in adjacent normal cells (14-16), which facilitates tumor cell escape from complement attack $(17,18)$. Although previous reports have described CD59 as a prognostic biomarker in breast cancer (19-21) playing important roles in immunotherapy of human malignancies (22), the role of CD59 in breast cancer growth and prognosis has not been fully elucidated.

\section{Materials and methods}

Ethical standards. The informed consent form was signed by each participant, and appropriate ethics committee approval was obtained. All the experiments complied with the current laws of China.

Cell preparation. The MDA-MB-231 breast carcinoma cell line was purchased from American Type Culture Collection (ATCC; Manassas, VA, USA). The MDA-MB-231-HM breast carcinoma cell line, which readily metastasizes to the lungs, was established previously (23). Briefly, MDA-MB-231 cells $\left(2 \times 10^{7}\right)$ were orthotopically injected into the exposed axillary mammary fat pad of athymic mice after anesthetization. Mice were sacrificed after 8-10 weeks under anesthetization and pulmonary metastatic lesions tissue samples were removed for primary culture in Leibovitz's L-15 (Gibco) medium supplemented with $10 \%(\mathrm{v} / \mathrm{v})$ fetal bovine serum (PAN Biotech) at $37^{\circ} \mathrm{C}$ in a humidified atmosphere containing $5 \% \mathrm{CO}_{2}$. Cells were passaged continuously until there were no apparent mesenchymal cells. Cells grown to $\sim 80 \%$ confluence were reinoculated into the mammary fat pad as described above and tissue samples from metastatic lesions were again obtained and treated as above. The procedures were repeated five to six times. A highly lung-metastatic variant of parental MDA-MB-231 cell line, termed MDA-MB-231-HM was established, followed by essential detections, such as Mycoplasma contamination. These two cell lines were cultured in Leibovitz's L-15 (Gibco) medium supplemented with $10 \%$ (v/v) fetal bovine serum (PAN Biotech) at $37^{\circ} \mathrm{C}$ in a humidified atmosphere containing $5 \% \mathrm{CO}_{2}$. The pLKO.1-TRC cloning vector (Addgene plasmid 10878) was utilized to construct two shRNA plasmids, shCD59 and shCD59', via insertion of shCD59F 5'-CCGGCCGTCA ATTGTTCATCTGATTCTCGAGAATCAGATGAACAATT GACGGTTTTTG-3' and shCD59R 5'-AATTCAAAAACC GTCAATTGTT CATCTGATTCTCGAGAATCAGATGAA CAATTGACGG-3' or shCD59'F 5'-CCGGGCTAACGTACT ACTGCTGCAACTCGAGTTGCAGCAGTAGTACGTTAGC T TTTTG-3' and shCD59'R 5'-AATTCAAAAAGCTAACGT ACTACTGCTGCAACTCGAGTTGCAGCAGTAGTACGTT AGC-3', respectively. Lentiviruses containing one of 2 control vectors or one of 2 CD59-targeting shRNAs were added to MDA-MB-231-HM culture medium with $8 \mu \mathrm{g} / \mathrm{ml}$ polybrene (Sigma-Aldrich), and the cells were incubated with the indicated lentiviruses for $24 \mathrm{~h}$. The infected cells were selected using $1 \mu \mathrm{g} / \mathrm{ml}$ puromycin (Sigma-Aldrich).

Antibodies and other reagents. Anti- $\beta$-actin (C4), anti-TFIIB (D-3), anti-CD59 (H-7), anti-CD46 (M177), anti-CD55 (H-7),
anti-phospho-ERK1/2 (E-4), anti-p65 (F-6), anti-p50 (E-10), anti-cRel (B-6), anti-Sp1 (E-3), anti-CREB-1 (24H4B), and antiphosphorylated CREB-1 (Ser-133) antibodies were purchased from Santa Cruz Biotechnology. Anti-Akt (10176-2-AP) was purchased from ProteinTech Group. Phospho-Akt ${ }^{\mathrm{Ser} 473}$ (2118-1) antibody was purchased from Epitomics. FITC-conjugated mouse anti-human CD59 mAb (p282/H19) was purchased from BD Pharmingen. A rabbit anti-CD59 polyclonal antibody (10742-1-AP) was obtained from Proteintech Group. Polybrene, puromycin, and CelLytic M (for mammalian cell lysis and extraction) were purchased from Sigma-Aldrich. Cell Counting Kit-8 reagents were purchased from Dojindo. U0126 and MK2206 were purchased from Selleck Chemicals and used at terminal concentrations of 1 and $2 \mu \mathrm{M}$.

Immunoblotting. Total cell lysates were extracted using a mammalian cell lysis/extraction reagent (Sigma-Aldrich). Nuclear and cytoplasmic proteins were isolated using a nuclear protein extraction kit (Active Motif). Immunoblotting was done, essentially as described (24). The primary antibodies used were as follows: anti- $\beta$-actin (C4; Santa Cruz Biotechnology), anti-TFIIB (D-3; Santa Cruz Biotechnology), anti-CD59 (H-7; Santa Cruz Biotechnology), anti-CD46 (M177; Santa Cruz Biotechnology), anti-CD55 (H-7; Santa Cruz Biotechnology), anti-ERK1/2 (C-9; Santa Cruz Biotechnology), anti-anti-phospho-ERK1/2 (E-4; Santa Cruz Biotechnology), anti-p65 (F-6; Santa Cruz Biotechnology), anti-p50 (E-10; Santa Cruz Biotechnology), anti-cRel (B-6; Santa Cruz Biotechnology), anti-Sp1 (E-3; Santa Cruz Biotechnology), anti-phosphorylated Sp1 (phospho-T453; Bioworld Technology), anti-CREB-1 (24H4B; Santa Cruz Biotechnology), anti-phosphorylated CREB-1 (Ser-133; Santa Cruz Biotechnology), rabbit anti-CD59 polyclonal antibody (10742-1-AP; ProteinTech Group). The quality of loading and transferring was assessed by immunostaining with the $\beta$-actin antibody. The dilutions for the antibodies were as follows: 1:500 for CD59, 1:500 for CD55, 1:500 for CD46, 1:1,000 for TFIIB, 1:1,000 for p65, 1:500 for p50 1:1,000 for cRel, 1:1,000 for Sp1, 1:500 for phosphorylated Sp1, 1:1,000 for CREB-1, 1:1,000 for phosphorylated CREB-1, 1:500 for ERK1/2, 1:500 for phospho-ERK1/2, 1:1,000 for rabbit anti CD59 polyclonal antibody and 1:2,000 for $\beta$-actin.

Cell proliferation analysis. CCK8 assays were used to measure cell proliferation. The indicated cells were seeded at a density of $2 \times 10^{3}$ cells in a volume of $200 \mu 1$ per well in 96-well plates (Nunc). Before detection, $100 \mu \mathrm{l}$ of fresh culture medium was added into each well after the cells had been washed twice with PBS. Then, $10 \mu 1$ of CCK8 solution (Dojindo) was added to each well, and the plates were incubated for 7 days at $37^{\circ} \mathrm{C}$. Cell viability was measured as the absorbance at $450 \mathrm{~nm}$ with a microplate reader (BioTek), and the mean optical density (OD450) values from triplicate wells for each cell line were used as an index of cell viability. All the experiments were performed in triplicate.

Flow cytometry analysis. CD59 expression at the cell membrane was detected by flow cytometry as described previously (24). Briefly, the harvested cells were incubated with a FITC-conjugated anti-human CD59 antibody 
(BD Pharmingen) and then analyzed using a Cytomics FC 500 MPL (Beckman Coulter).

For cell cycle analysis, cells were digested into single-cell suspension and $1 \times 10^{6}$ cells were suspended by $1 \mathrm{ml}$ PBS. Three milliliters Pre-cold ethanol $(3 \mathrm{ml})$ at $-20^{\circ} \mathrm{C}$ was added into cell suspension with vortex and then the sample was fixed at $-20^{\circ} \mathrm{C}$ overnight. Fixed cells were collected by centrifugation at 2,000 rpm for $5 \mathrm{~min}$ and the supernatant was discarded. Cells were suspended by PBS for $15 \mathrm{~min}$ at room temperature. Cells were then recollected by centrifugation at $800 \mathrm{rpm}$ for $5 \mathrm{~min}$ and stained by Cell Cycle Staining kit [MultiSciences (Lianke) Biotechnology] as instructed. Then cell cycle distribution was analyzed by flow cytometry.

Quantitative real-time PCR. Total RNA was extracted from cells with or without relative treatments using TRIzol reagent (Invitrogen) and reverse transcribed into cDNA using PrimeScript $^{\mathrm{TM}}$ RT Master Mix (Takara) following the manufacturer's instructions. Standardized cDNA was used as template for amplification of 40 cycles with SYBR Green Master Mix and specific primers of different genes on ABI PRISM 7900HT (Applied Biosystems). $\beta$-actin was used as endogenous control, and samples were analyzed in triplicate. The primers for amplification of $\beta$-actin were 5'-ACC GAGCGCGGCTACAG-3' (forward) and 5'-CTTAATGTCA CGCACGATTTCC-3' (reverse). The primers for amplification of CD59 were 5'-ATGGGAATCCAAGGAGGGTC-3' (forward) and 5'-ATTGACGGCTGTTTTGCAGT-3' (reverse).

Colony formation assay. Single-cell suspension was obtained by $0.25 \%$ trypsin digestion and 500 cells were seeded in each well of 6-well plates. After incubation at an atmosphere of $5 \% \mathrm{CO}_{2}$ at $37^{\circ} \mathrm{C}$ for 15 days, cells were washed with PBS twice and fixed with $4 \%$ paraformaldehyde/PBS (Solarbio) for $30 \mathrm{~min}$. Then cells were stained by $0.1 \%$ crystal violet staining solution (Solarbio) for $30 \mathrm{~min}$ and washed with PBS 3 times. The number of colonies was counted and the colony formation efficiency was calculated as number of colonies/number of cells seeded x $100 \%$.

Immunohistochemistry(IHC) staining. Immunohistochemistry for CD59, Akt, and phospho-Akt was performed on the slides, using a two-step proto-col (EnVision ${ }^{\mathrm{TM}}$ Detection Systems, Dako, Glostrup, Denmark). The slides were dewaxed with xylene, gradually hydrated with gradient ethanol, and washed with phosphate-buffered saline (PBS). Antigens were retrieved by boiling the slides in citrate buffer $\left(\mathrm{pH} \mathrm{6.0)}\right.$ ) at $121^{\circ} \mathrm{C}$ for $15 \mathrm{~min}$. Endogenous peroxidase was blocked by incubating the slides in $3 \% \mathrm{H}_{2} \mathrm{O}_{2}$ solution. Then, the slides were washed in PBS for $10 \mathrm{~min}$, blocked with $10 \%$ normal goat serum (CW0130; CWBIO, Beijing, China) for $30 \mathrm{~min}$ at $37^{\circ} \mathrm{C}$, and incubated with anti-CD59 (H-7; Santa Cruz Biotechnology) at $4^{\circ} \mathrm{C}$ overnight. The antibody dilution was 1:100 for the CD59 antibodies and 1:100 for Akt and phospho-Akt antibody. The immunoreactive products were visualized by the catalysis of 3,3-diaminobenzidine by horseradish peroxidase (EnVision ${ }^{\mathrm{TM}}$ Detection Systems), following extensive washing. The slides were then counterstained with Gill hematoxylin (BA-5021, Zhuhai, China) and dehydrated in ascending grades of ethanol, and finally, cleared in xylene and mounted under a coverslip.

Evaluation of immunohistochemistry analysis. As in our study, the expression of CD59 and phospho-Akt was semi-quantitatively classified according to the staining index (SI; range 0-9), which was calculated by multiplying the staining intensity by the proportion score. The staining intensities were classified according to four grades ( 0 denoting negative; 1 , weak; 2 , moderate; and 3, strong), and the proportion score was graded as the percentage of cells that were stained ( 0 denoting 0 to $<10 \%$ of cells; 1 , between 10 and $30 \%$ of cells; 2 , between 31 and $60 \%$ of cells; and 3 , staining of $>60 \%$ of cells). In this study, SI 3 was defined as CD59-positive staining, whereas SI $<3$ was defined as negative staining. The IHC scores were independently determined by two experienced pathologists (Yiqun Du and Yun Xia) as described previously with slight modifications $(25,26)$.

Xenograft animal studies. All the mouse experiments were performed according to a protocol approved by the Fudan University Shanghai Cancer Center (Shanghai Cancer Center Ethics Committee) Animal Care and Use Committee. Female Balb/C nude mice, aged 8 weeks, were purchased from SLAC Laboratory Animals, and were maintained under specific pathogen-free conditions. The mice were acclimated in-house for $\geq 7$ days prior to use. A total of $1.0 \times 10^{7}$ cancer cells were suspended in complete culture medium containing 10\% FBS and then injected into the left fourth mammary fat pad of each female BALB/c nude mouse; a total of seven mice were included in each group. Tumor size was measured twice weekly with calipers, and tumor volume was calculated using the following formula: (width) ${ }^{2} \mathrm{x}$ length/2. At the endpoint of the experiment, the tumor tissues were carefully excised and weighed after the mice were anesthetized and sacrificed with $\mathrm{CO}_{2}$.

Collection of human breast cancer specimens. Between May 2008 and July 2008, 120 women with breast cancer, as confirmed by core needle biopsy, with no prior treatment were treated by surgical resection at the Shanghai Cancer Center, Fudan University. This study was approved by the independent ethics committee/institutional review board of the Fudan University Shanghai Cancer Center (Shanghai Cancer Center Ethics Committee). All patients provided written informed consent before they were included in this study.

Statistical analysis. All the data are presented as the mean and standard deviation (SD). The $\chi^{2}$ test was used to evaluate the relationship between patient characteristics and body mass index (BMI) and to compare groups based on CD59 expression. Fisher's exact test was performed when necessary. A multivariate logistic regression model to predict metastasis was utilized based on both categorical variables (CD59 expression, tumor size, lymph node status, cancer embolus, ER status, PR status, and HER-2 status) and continuous variables (age) that were evaluated at the time of diagnosis. All the statistical tests were two-sided, and P-values $<0.05$ were considered significant. All the analyses were performed using SPSS (version 13.0; SPSS Inc., Chicago, IL, USA). 


\section{Results}

MDA-MB-231-HM cells express higher levels of CD59, but not of CD46 or CD55, than MDA-MB-231 cells, potentially via the ERK signaling pathway. We performed immunoblotting assays with total cell lysates to elucidate the expression levels of CD59 in breast cancer cell lines, such as ZR-75-30, T47D, MCF-7, MDA-MB-453, SK-BR-3, ZR-75-1, MDA-MB-468, MDA-MB-231 cells and the derived MDA-MB-231-HM cells, which are prone to lung metastasis. CD59 expression was significantly upregulated in MDA-MB-231-HM cells and ZR-75-30 cells (Fig. 1A). CD59, but not that of two other mCRPs (CD46 and CD55) was upregulated in MDA-MB-231-HM compared with MDA-MB-231 (Fig. 1B). Flow cytometry assays and real-time PCR further confirmed that CD59 expression was significantly higher in MDA-MB-231-HM cells compared to the parental cells (Fig. 1C and D).

We recently reported that the constitutive expression of CD59 is controlled by $\mathrm{Sp} 1$, whereas the inducible expression of CD59 is regulated by NF- $\mathrm{KB}$ and enhancer-binding CREB (24). To investigate the underlying mechanisms by which MDA-MB-231-HM cells upregulate CD59, we first examined the nuclear levels of total and phosphorylated $\mathrm{Sp1}$ and the nuclear translocation of NF-kB. There were no significant differences between these two cell lines with respect to $\mathrm{Sp} 1$ and NF- $\kappa B$ signaling (Fig. 1E). However, there was increased phosphorylation of CREB, another transcription regulator, in MDA-MB-231-HM cells compared to MDA-MB-231 cells (Fig. 1F). CREB can be directly or indirectly phosphorylated by phospho-Akt and phospho-ERK (27-29), so we determined the levels of Akt and ERK phosphorylation. Both phospho-Akt and phospho-ERK were significantly increased in MDA-MB-231-HM cells compared to MDA-MB-231 cells (Fig. 1G). The same result failed to apply to the other group of breast cancer cells: ZR-75-1 and ZR-75-30 (data not shown), as the levels of phosphorylated CREB between ZR-75-1 and ZR-75-30 showed no significant difference. This is understandable considering the complicated regulation of CD59 and that MDA-MB-231-HM was derived from MDA-MB-231. To investigate the mechanism underlying the upregulation of CD59 in cell MDA-MB-231-HM, we inhibited ERK phosphorylation and Akt phosphorylation using U0126 and MK2206, respectively. The inhibition of ERK phosphorylation downregulated CD59 expression both at mRNA level and protein level (Fig. 1H and I), while the inhibition of Akt phosphorylation failed (Fig. 1J). These findings suggested that CD59 was upregulated in breast cancer MDA-MB-231-HM cells and that this upregulation may occur via the ERK pathway.

CD59 knockdown suppresses the tumor growth of MDA-MB231-HM cells. Breast cancer lung metastasis leads to a poor prognosis $(6,7)$. We utilized MDA-MB-231-HM cells, which are prone to lung metastasis, to explore the role of CD59 in the highly metastatic MDA-MB-231-HM cells. We knocked down CD59 expression in MDA-MB-231-HM cells using two vectors expressing shRNA against CD59, referred to as 231-HM-shCD59 and 231-HM-shCD59' (the corresponding controls were designated 231-HM-Vector and 231-HM-Vector', respectively). As shown in Fig. 2A, CD59 was robustly knocked down in MDA-MB-231-HM cells. 231-HM-shCD59 was more efficient at knocking down CD59 expression, and we therefore selected this construct for use in subsequent studies.

We then used CCK 8 assays to determine the effect of CD59 knockdown on cell proliferation. As shown in Fig. 2B, 231-HM-shCD59 cells lacking CD59 expression grew more slowly than 231-HM-Vector cells beginning on day 4 after cell plating $(\mathrm{P}<0.01)$. Colony formation assay showed similar result $(\mathrm{P}<0.01)$ (Fig. $2 \mathrm{C})$, and cell cycle analysis indicated that this growth arrest may due to the cell cycle arrest in 231-HM-shCD59 cells (Fig. 2D). We next implanted 231-HM-Vector and 231-HM-shCD59 cells into the left fourth mammary fat pads of female athymic nude mice. Primary tumors developed successfully in all mice, and CD59 knockdown markedly decreased tumor growth, as evidenced by tumor size (Fig. $2 \mathrm{E}, \mathrm{P}<0.02, \mathrm{P}<0.01$ ). At 5 weeks after injection, all the mice were sacrificed, and their tumors and lungs were resected, as MDA-MB-231-HM is prone to lung metastasis. The tumors were weighed, and the lungs were examined by hematoxylin and eosin (H\&E) staining. The tumors in the 231-HM-shCD59-injected mice were clearly much smaller than those in the control mice, and the corresponding tumor weights were consistent with this observation (representative images are shown in Fig. 2F, $\mathrm{P}<0.01)$. In the $\mathrm{H} \& \mathrm{E}$-stained slices, we found metastases in 3/10 lungs from the 231-HM-Vector group, whereas none of the 10 lungs from the 231-HM-shCD59 group had metastatic lesions; representative images are shown in Fig. 2G. These data indicated that CD59 knockdown suppressed cell proliferation both in vitro and in vivo and may decrease lung metastasis in vivo.

High CD59 expression levels were associated with lung metastasis and poor prognosis in breast cancer patients. To explore the clinical significance of CD59 expression in prognosis, we analyzed a cohort of 120 breast cancer patients, 58 with lung metastases and 62 without metastatic disease. We performed IHC staining for CD59 expression, and the expression scores were ranked by two independent pathologists who were blinded to the identity of each sample. Representative images of CD59 in 3 patients with metastasis and in 3 patients without metastasis are shown in Fig. 3. We retrospectively collected complete clinical data, including age, tumor size, pathological assessment and follow-up visit details. Table I shows the characteristics of the patients grouped based on CD59 expression. Only the presence of metastasis was significantly associated with CD59 expression $(\mathrm{P}=0.001)$. There was a higher percentage of HER-2-negative patients among those with high CD59 expression. However, age, tumor size, node status, tumor histology, ER status, PR status and HER-2 status were not significantly correlated with CD59 expression.

The metastasis rate was $48.3 \%$ (58/120) in the study cohort. Table II shows the univariate and multivariate analyses of the metastasis rate predictors using a logistic regression model. In the univariate model, there were no significant relationships between metastasis rate and tumor size, tumor histology, ER status, PR status or HER-2 status $(P>0.05)$. However, metastasis was significantly associated 
A
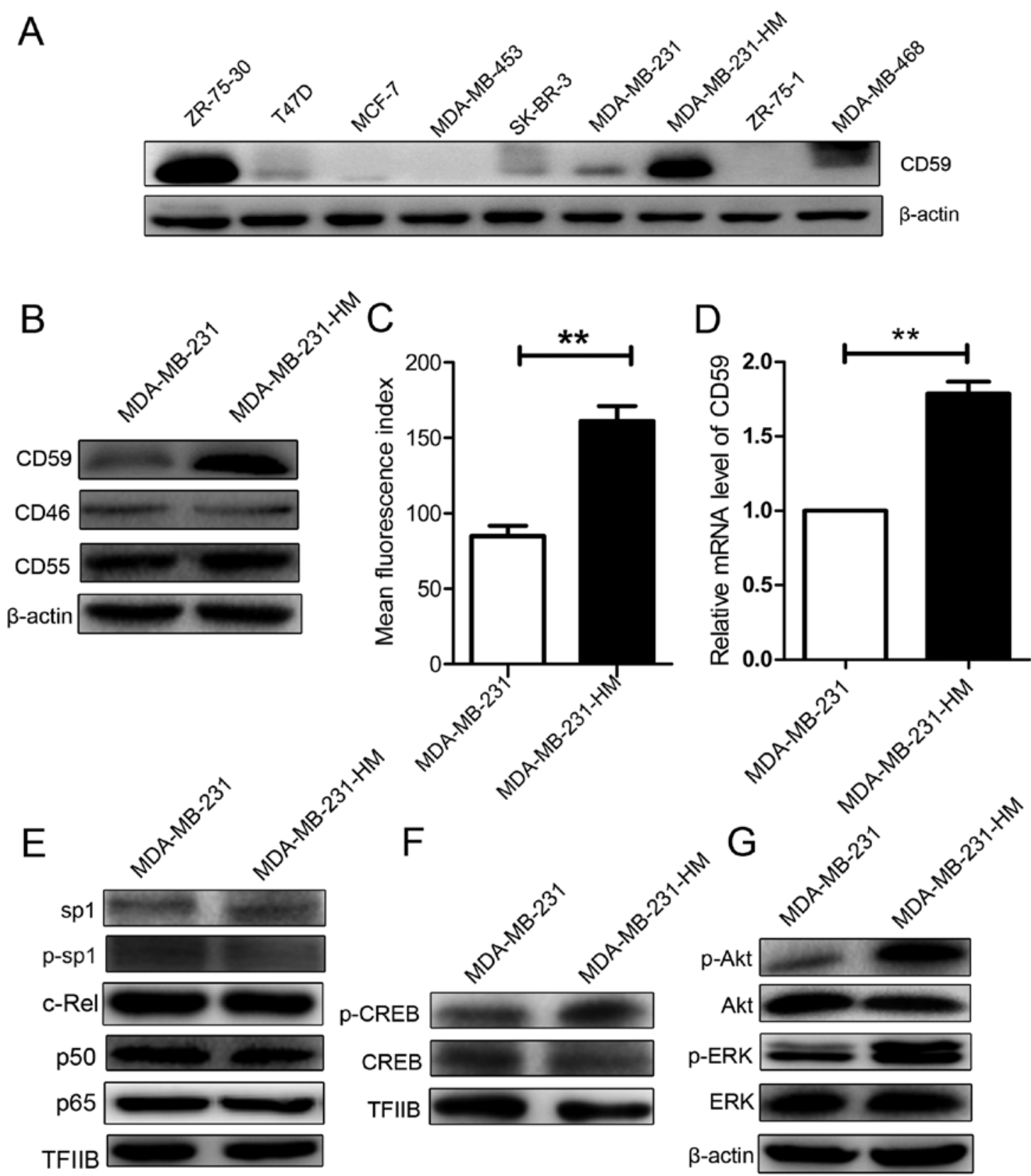

$x^{2} \mathrm{C}$
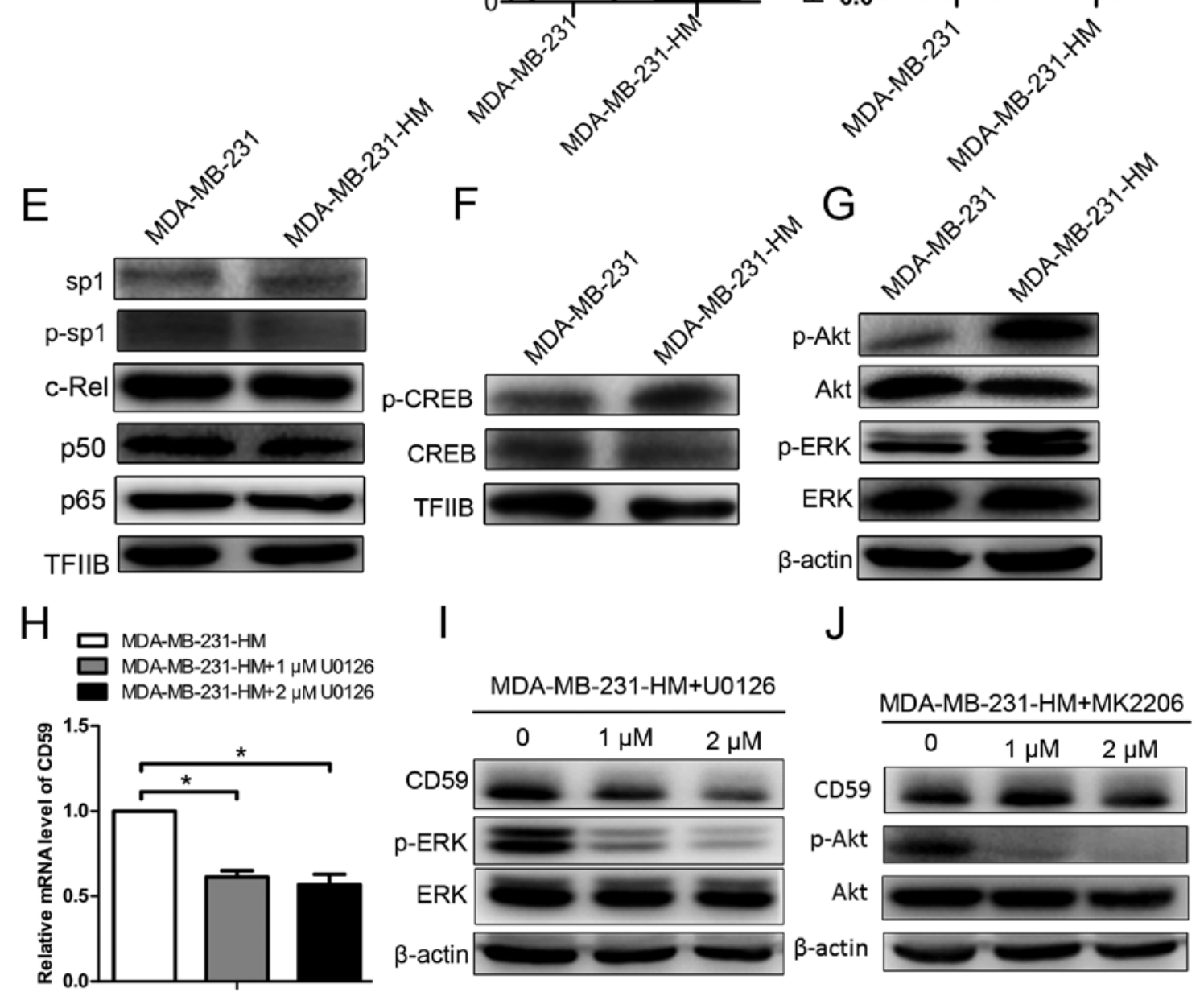

Figure 1. CD59 is overexpressed in MDA-MB-231-HM cells compared to MDA-MB-231 cells. (A) CD59 was upregulated in MDA-MB-231-HM cells and ZR-75-30 cells. CD59 expression was detected in different cell lines by immunoblotting. (B) CD59, but not the other mCRPs (CD46 and CD55), was highly expressed in MDA-MB-231-HM cells as detected by immunoblotting of total cell lysates. (C and D) Flow cytometry assays and real-time PCR further verified the upregulation of CD59 in MDA-MB-231-HM cells. CD59 expression levels were measured using a FITC-conjugated anti-CD59 antibody in flow cytometry assays, and relative mRNA level was measured by real-time PCR. The mean \pm SD was derived from 3 independent experiments; ${ }^{* *} \mathrm{P}<0.01$. (E and F) The levels of trans-acting factors involved in CD59 transcription were detected in nuclear extracts. (G) Immunoblotting assays were used to detect the phosphorylation of Akt and ERK, two signaling molecules that are upstream of CREB. (H-J) MDA-MB-231-HM cells were treated with U0126 or MK2206 at concentrations of 1 and $2 \mu \mathrm{M}$ for $24 \mathrm{~h}$ and then the phosphorylation of ERK and Akt, total ERK and Akt and CD59 were detected by immunoblotting in cells treated with U0126 and MK2206, and mRNA level of CD59 was detected in cells treated with U0126. 
A

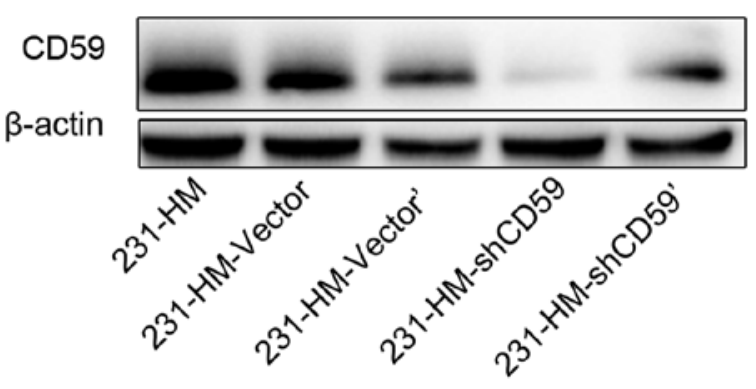

B

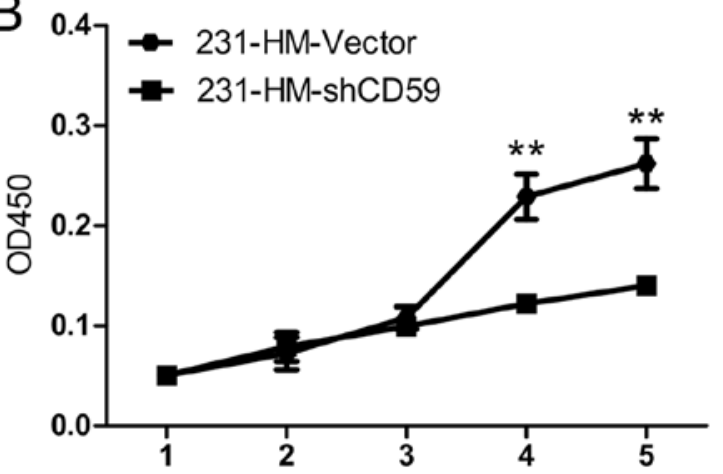

C

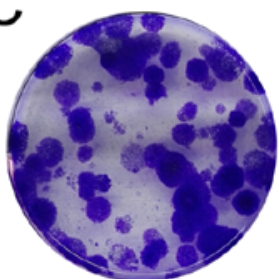

231-HM-shCD59

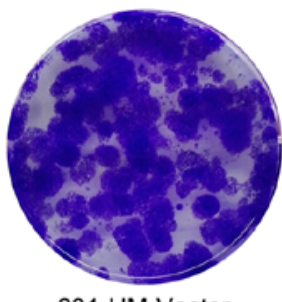

231-HM-Vector

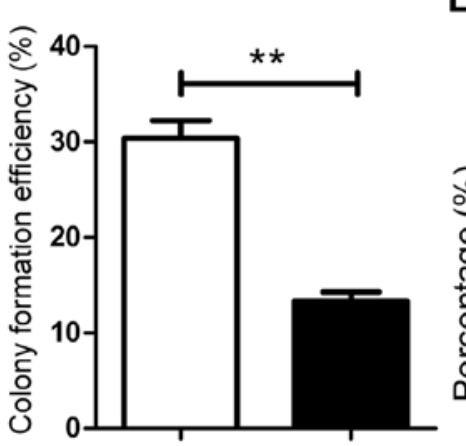

D
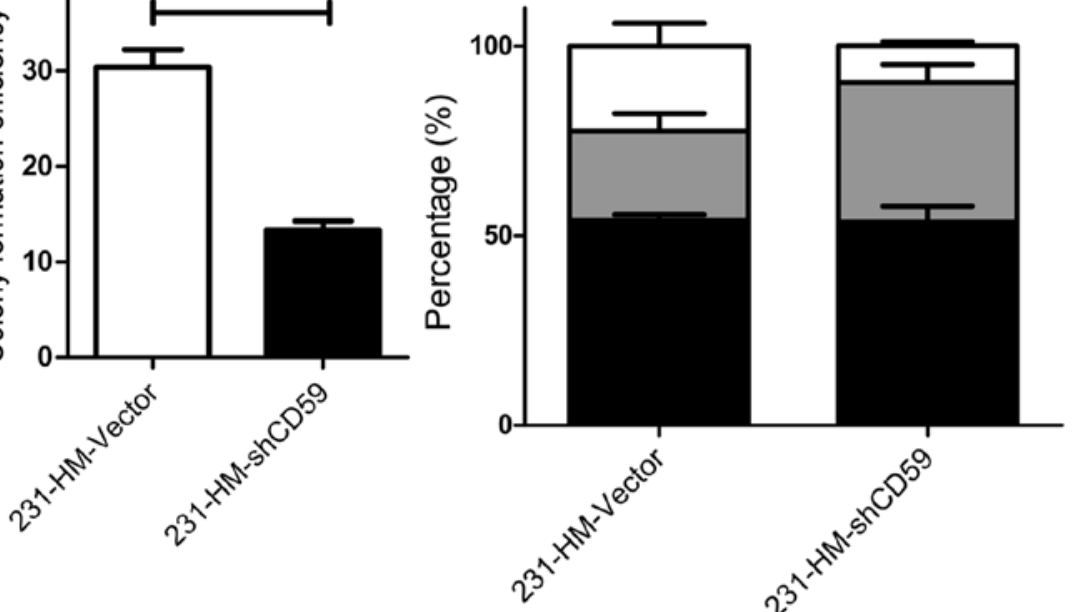

G1

$\square \mathrm{S}$

$\square$ G2/M

$\mathrm{F}$

E

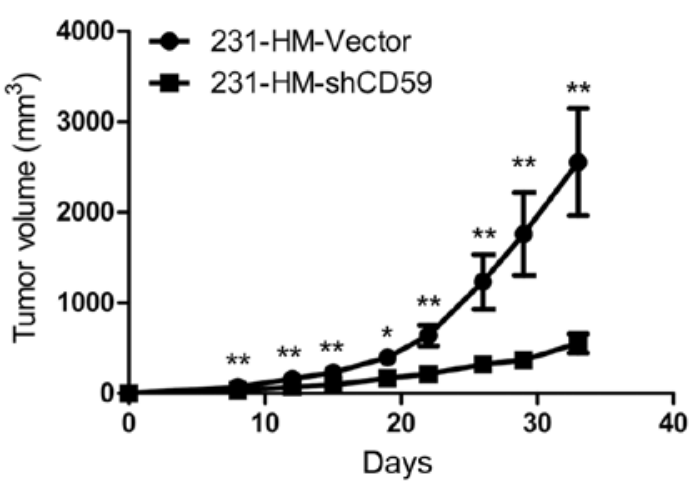

G

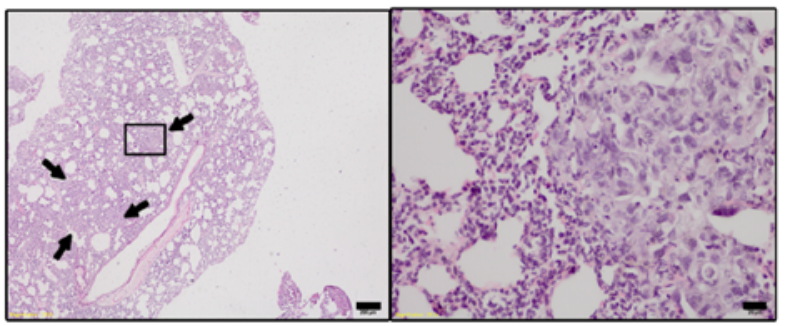

231-HM-Vector
231-HM-shCD59

231-HM-Vector
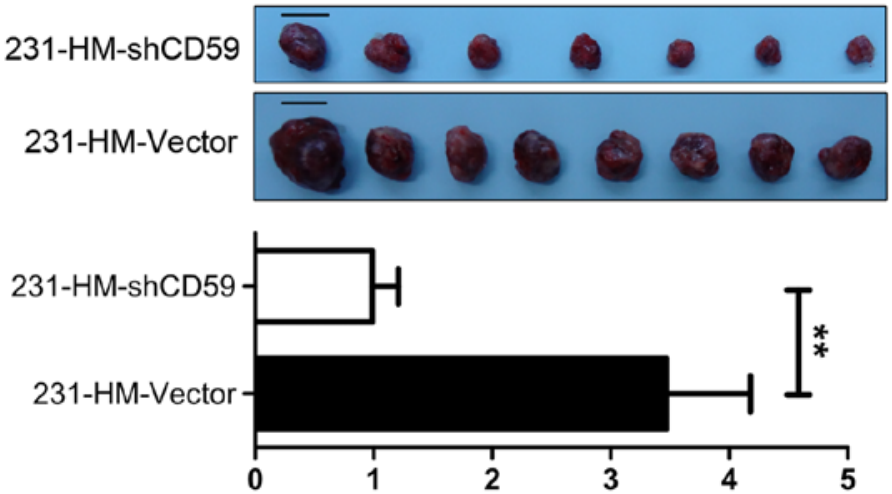

Tumor weight (g)

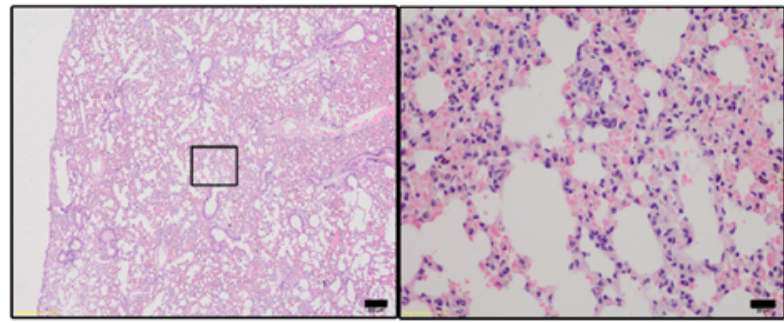

231-HM-shCD59

Figure 2. CD59 knockdown suppresses the cell proliferation and lung metastasis of MDA-MB-231-HM cells. (A) The efficacy of specific shRNAs against CD59 in different vectors was evaluated by immunoblotting. (B) CD59 knockdown in MDA-MB-231-HM cells suppressed cell proliferation in vitro as measured by CCK8 assays. Mean \pm SD, $n=3$. ${ }^{* *} \mathrm{P}<0.01$. (C) CD59 knockdown inhibited cell proliferation as detected by colony formation assay. Five hundred cells of each stable cell pool were plated and colony formation efficiency was calculated. (D) CD59 knockdown lead to cell cycle arrest according to cell cycle staining assay. (E and F) CD59 insufficiency in MDA-MB-231-HM cells decreased tumor growth in the xenografted nude mice. (E) Tumor volume at different time-points. ${ }^{*} \mathrm{P}<0.02,{ }^{* *} \mathrm{P}<0.01$; (F) Tumor images (top) and the calculated tumor weights (bottom) at the study end-point. ${ }^{* *} \mathrm{P}<0.01$. Scale bars, 2 cm. (G) Representative H\&E staining of lungs from mice implanted with control or CD59-knockdown MDA-MB-231-HM cells. The arrow indicates metastatic breast cancer cells. Scale bars, $200 \mu \mathrm{m}$ in the left panels and $20 \mu \mathrm{m}$ in the right panels within each group. 

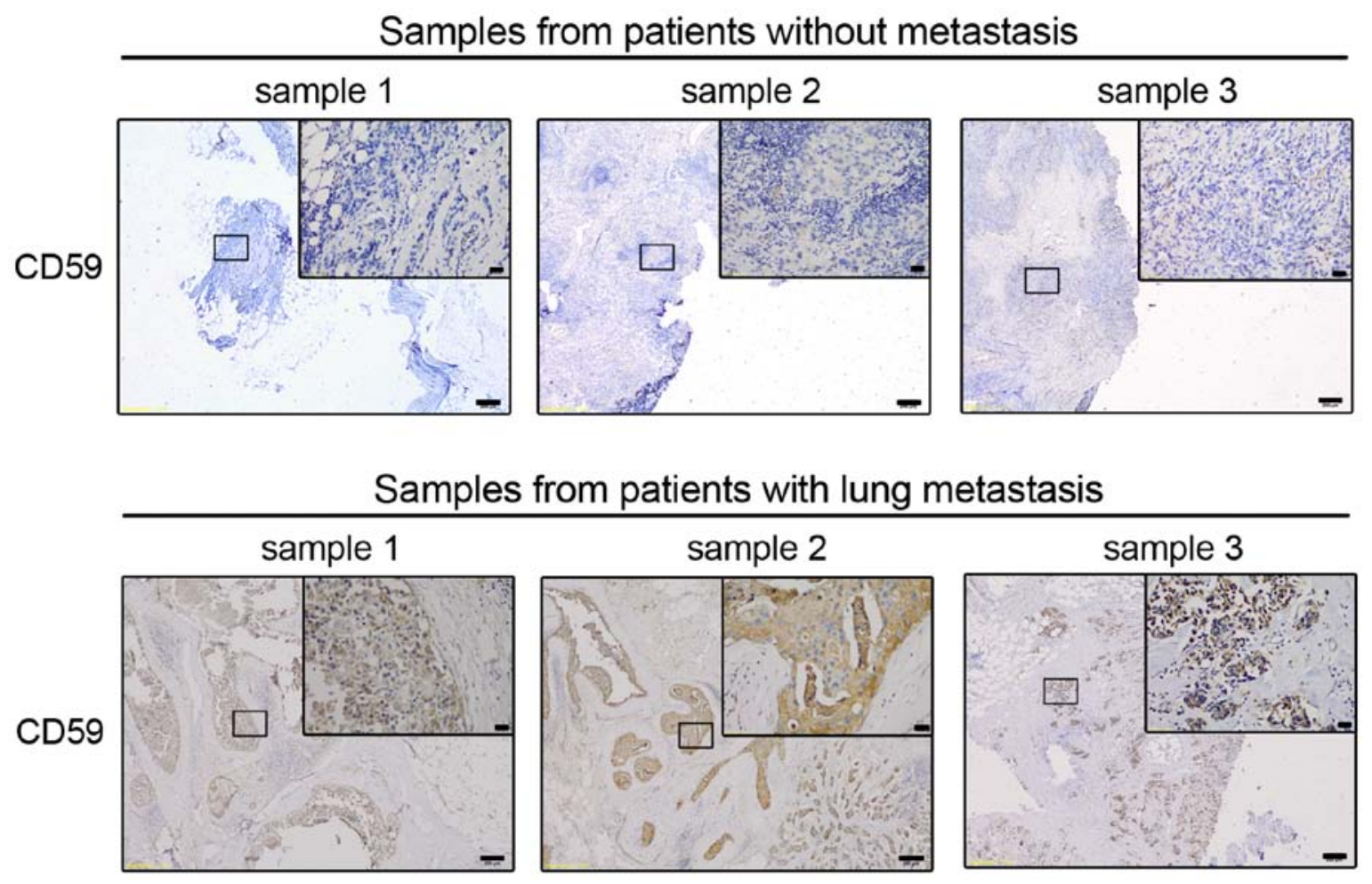

Figure 3. CD59 was highly expressed in patients with lung metastases compared to patients without metastatic disease. Three representative IHC stains of CD59 expression in breast cancer specimens from patients with or without metastasis. Scale bar, $200 \mu \mathrm{m}$ in the large rectangle and $20 \mu \mathrm{m}$ in the small rectangle.

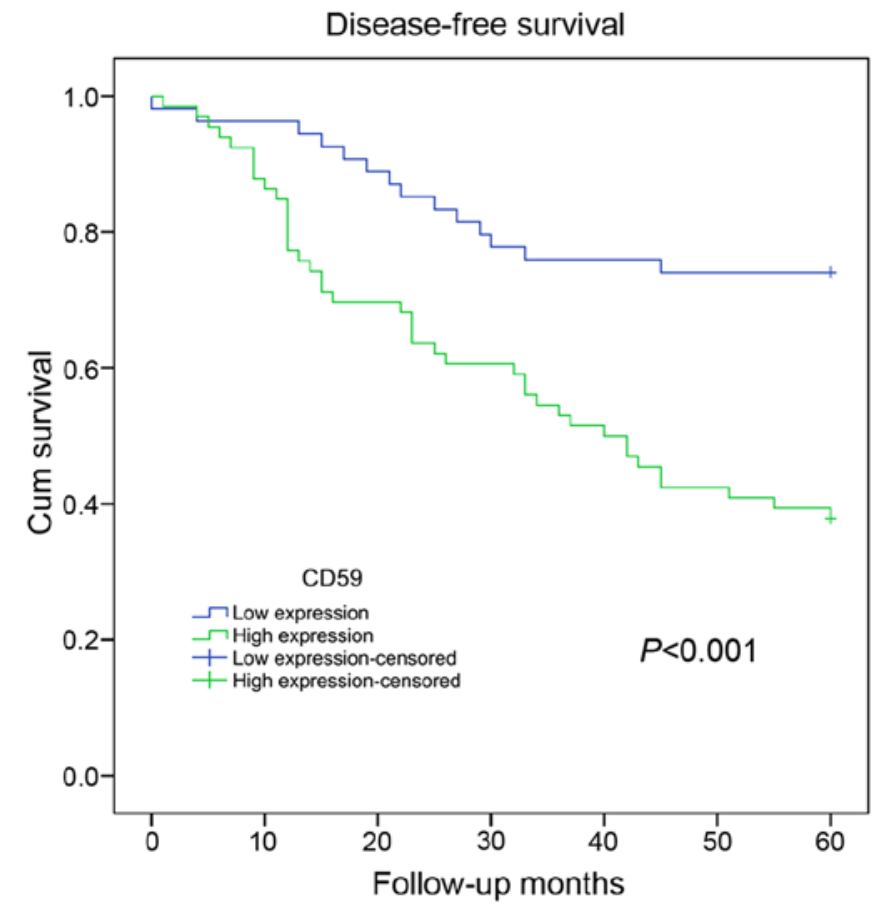

Figure 4. Upregulated CD59 expression detected by IHC staining is associated with poor prognosis in breast cancer patients. Kaplan-Meier curves depicting disease-free survival based on an analysis of 120 breast cancer patients (58 with metastasis and 62 without metastasis).

with age, lymph node status, cancer embolus and CD59 expression $(\mathrm{P}<0.05)$. Patients 35 years or older, as well as those with positive lymph node status, cancer embolus or high
CD59 expression were more likely to develop metastases. In the multivariate model, CD59 expression, lymph node status and cancer embolus were independent predictors of metastasis, with P-values of $0.041,0.032$ and 0.008 , respectively. Notably, a Kaplan-Meier survival analysis revealed that CD59 expression levels were negatively correlated with relapse-free survival among breast cancer patients $(\mathrm{P}<0.001)$. The analysis indicated that patients with higher CD59 expression would have a worse prognosis compared to patients with lower CD59 expression (Fig. 4). These clinical data suggested that CD59 expression may be a prognostic biomarker for metastasis and outcome in breast cancer patients.

\section{Discussion}

Human CD59 is a $18-20-\mathrm{kDa}$ membrane complement regulatory protein (mCRP) that is anchored to the cell membrane by glycophosphatidylinositol (GPI) and protects innocent host cells from activated complement destruction (15). In most solid tumors, CD59 is overexpressed compared to adjacent normal cells, and inhibiting CD59 function can improve breast cancer treatment $(14,30,31)$. Distant metastases are lethal to breast cancer patients (7). We hypothesized that CD59 may protect tumor cells from complement attack, thus facilitating breast cancer metastasis, leading to poor prognosis. Because lung metastasis is unequivocally a clinical challenge in breast cancer treatment and leads to poor prognosis in breast cancer patients $(6,7)$, we utilized the MDA-MB-231-HM cell line to elucidate the roles of mCRPs; MDA-MB-231-HM cells are more prone to lung metastasis than parental MDA-MB-231 cells. We discovered that among the three mCRPs, only 
Table I. Results of the correlation analysis of CD59 expression in 120 patients with breast cancer.

\begin{tabular}{|c|c|c|c|c|}
\hline Characteristic & $\begin{array}{c}\text { Patients } \\
\text { (no.) }\end{array}$ & $\begin{array}{c}\text { CD59 } \\
\text { low }\end{array}$ & $\begin{array}{l}\text { CD59 } \\
\text { high }\end{array}$ & P-value \\
\hline \multicolumn{5}{|l|}{ Age } \\
\hline$\leq 35$ & 13 & 6 & 7 & \multirow[t]{2}{*}{$0.578^{\mathrm{a}}$} \\
\hline$>35$ & 107 & 48 & 59 & \\
\hline \multicolumn{5}{|l|}{ Size $(\mathrm{cm})$} \\
\hline$<2$ & 21 & 7 & 14 & \multirow[t]{3}{*}{$0.139^{a}$} \\
\hline$\geq 2,<5$ & 90 & 45 & 45 & \\
\hline$\geq 5$ & 9 & 2 & 7 & \\
\hline \multicolumn{5}{|l|}{ Grade } \\
\hline I-II & 81 & 35 & 46 & \multirow[t]{2}{*}{$0.354^{\mathrm{a}}$} \\
\hline III & 39 & 19 & 20 & \\
\hline \multicolumn{5}{|l|}{$\begin{array}{l}\text { Lymph node } \\
\text { status }\end{array}$} \\
\hline- & 76 & 38 & 38 & \multirow[t]{2}{*}{$0.104^{\mathrm{a}}$} \\
\hline+ & 44 & 16 & 28 & \\
\hline \multicolumn{5}{|l|}{ ER } \\
\hline- & 52 & 19 & 33 & \multirow[t]{2}{*}{$0.074^{\mathrm{a}}$} \\
\hline+ & 68 & 35 & 33 & \\
\hline \multicolumn{5}{|l|}{ PR } \\
\hline- & 68 & 27 & 41 & \multirow[t]{2}{*}{$0.126^{\mathrm{a}}$} \\
\hline+ & 52 & 27 & 25 & \\
\hline \multicolumn{5}{|l|}{ Her-2 } \\
\hline- & 91 & 39 & 52 & \multirow[t]{2}{*}{$0.267^{\mathrm{a}}$} \\
\hline+ & 28 & 15 & 14 & \\
\hline \multicolumn{5}{|c|}{ Cancer embolus } \\
\hline- & 84 & 41 & 43 & \multirow[t]{2}{*}{$0.140^{\mathrm{a}}$} \\
\hline+ & 36 & 13 & 23 & \\
\hline \multicolumn{5}{|l|}{ Metastasis } \\
\hline- & 65 & 40 & 25 & \multirow[t]{2}{*}{$0.001^{\mathrm{a}}$} \\
\hline+ & 55 & 14 & 41 & \\
\hline
\end{tabular}

The bold values are significant $(\mathrm{P}<0.05)$. ER, estrogen receptor; $\mathrm{PR}$, progesterone receptor; Her-2, human epidermal growth factor receptor-2. ${ }^{\text {aPearson's }} \chi^{2}$ test or two-sided Fisher's exact test.
CD59 was upregulated in MDA-MB-231-HM cells. After knocking down CD59 with a specific shRNA, we surprisingly found that MDA-MB-231-HM cell growth was significantly suppressed both in vitro and in vivo. CD59 knockdown in MDA-MB-231-HM cells inhibited the formation of metastatic lesions in the orthotopic xenograft model, although it was not statistically significant. We also determined that CD59 overexpression was associated with poor prognosis in a cohort of 120 breast cancer patients. We first demonstrated that CD59 was associated with MDA-MB-231-HM cell growth in vitro and in vivo and then correlated CD59 levels with breast cancer poor prognosis.

CD59 transcriptional regulation involves many pathways (24), including p-CREB, as confirmed by Du et al in our laboratory research group, by performing the CHIP assays. In this study, we showed that CD59 upregulation in MDA-MB-231-HM cells is related to p-CREB, furthermore, CD59 upregulation in MDA-MB-231-HM cells may result from ERK pathway activation which plays critical roles in cancer (32); one of its functions may be to upregulate CD59 through CREB (24,27-29). The protective roles of mCRPs, especially CD59, may facilitate tumor cell evasion from complement attack; the mCRPs are therefore obstacles to antibody-based immunotherapy for cancer $(13,14)$. In addition, CD59 overexpression protects MCF-7 breast cancer cells from complement-mediated cytolysis and promotes MCF-7 cell proliferation by inhibiting BCL-2 expression; conversely, CD59 knockdown upregulates Fas and caspase-3 to induce apoptosis (20). Besides, CD59 silencing in NSCLC cancer cells can enhance complement-mediated cell apoptosis and inhibit the growth of NSCLC (16), which shows accordance with our study.

Inspiring studies by Madjd et al (19) and Terp et al (21) demonstrated that the loss of CD59 expression in breast tumors correlates with poor survival (19), and that CD59 is inversely correlated with metastatic capability (21). Here, we showed that CD59 overexpression was associated with MDA-MB-231-HM cell growth as well as with poor outcome for breast cancer patients. Though CD59 knock-

Table II. Results of the univariate and multivariate analyses of CD59 expression in 120 patients with breast cancer.

\begin{tabular}{|c|c|c|c|c|c|c|c|c|}
\hline \multirow[b]{3}{*}{ Variable } & \multicolumn{4}{|c|}{ Univariate analysis } & \multicolumn{4}{|c|}{ Multivariate analysis } \\
\hline & \multirow[b]{2}{*}{ HR } & \multicolumn{2}{|c|}{$95 \% \mathrm{CI}$} & \multirow[b]{2}{*}{ P-value } & \multirow[b]{2}{*}{ HR } & \multicolumn{2}{|c|}{$95 \% \mathrm{CI}$} & \multirow[b]{2}{*}{ P-value } \\
\hline & & Lower & Upper & & & Lower & Upper & \\
\hline Age & 0.962 & 0.937 & 0.988 & 0.004 & 0.982 & 0.957 & 1.007 & 0.163 \\
\hline Tumor size & 1.148 & 0.622 & 2.117 & 0.659 & & & & \\
\hline ER & 0.692 & 0.408 & 1.176 & 0.174 & & & & \\
\hline PR & 0.799 & 0.466 & 1.371 & 0.415 & & & & \\
\hline Her-2 & 1.572 & 0.887 & 2.787 & 0.121 & & & & \\
\hline Grade & 1.612 & 0.940 & 2.766 & 0.083 & & & & \\
\hline Lymph node status & 4.793 & 2.746 & 8.366 & 0.000 & 2.270 & 1.033 & 4.987 & 0.041 \\
\hline Cancer embolus & 4.868 & 2.828 & 8.379 & 0.000 & 2.312 & 1.074 & 4.980 & 0.032 \\
\hline CD59 & 3.074 & 1.673 & 5.648 & 0.000 & 2.328 & 1.253 & 4.326 & 0.008 \\
\hline
\end{tabular}

The bold values are significant $(\mathrm{P}<0.05)$. ER, estrogen receptor; PR, progesterone receptor; HR, hazard ratio; Her-2, human epidermal growth factor receptor-2. 
down suppressed cell proliferation both in vitro and in vivo, there were no significant relationships between CD59 and tumor size in our clinical investigation. Corresponding to the clinical-tumor size does not present positive correlation with the prognosis of breast cancer. In the large study involving 520 patients reported by Madjd et al (19), lung metastasis was not evaluated, whereas we focused on lung metastasis and established a cohort of 120 patients with or without lung metastasis. In addition, in our study, a greater proportion of HR-negative and Her-2-negative patients had high CD59 expression. These differences may explain the discrepant conclusions. Terp et al (21) showed that CD59 is 'associated' with the aggressiveness of metastasis rather than metastasis colonization per se. This may be due to the inhibition of cell growth; in our study, CD59 knockdown resulted in a better 'outcome' for the mice, so the failure to observe metastatic lesions may due to cell growth inhibition during our experimental time. However, it has been reported that CD59 can mediate signal transmission through the Src family member Lck in T cells (33). We cannot eliminate the possibility that in MDA-MB-231-HM cells, complex signals may be transmitted by CD59 to promote cell proliferation and metastasis.

As a biomarker in breast cancer, CD59 may play versatile roles. This study revealed that CD59 overexpression may be a prognostic factor for poor survival in breast cancer patients. However, the mechanism of action of CD59 and its controversial roles in breast cancer, perhaps through $\mathrm{Src}$ family members or other signaling pathways, remain to be elucidated. Besides, as an immunotherapy 'candidate', CD59 may have other than the known roles in breast cancer treatment (22).

\section{Acknowledgements}

This study was supported by grants from the National Natural Science Foundation of China (81201531), the 2012 Shanghai Committee of Science and Technology Funds (12ZR1406200, 12DZ2260100 and 12140901502), and the Shanghai Committee of Science and Technology Fund for 2013, Qimingxing Project (11QA1401400 to X. Hu). We also would like to thank American Journal Experts editorial team for their language polish.

\section{References}

1. Siegel R, Ma J, Zou Z and Jemal A: Cancer statistics, 2014. CA Cancer J Clin 64: 9-29, 2014

2. Elmore JG, Armstrong K, Lehman CD and Fletcher SW: Screening for breast cancer. JAMA 293: 1245-1256, 2005.

3. Tabár L, Vitak B, Chen HH, Duffy SW, Yen MF, Chiang CF, Krusemo UB, Tot T and Smith RA: The Swedish Two-County Trial twenty years later. Updated mortality results and new insights from long-term follow-up. Radiol Clin North Am 38: 625-651, 2000

4. Soerjomataram I, Louwman MW, Ribot JG, Roukema JA and Coebergh JW: An overview of prognostic factors for long-term survivors of breast cancer. Breast Cancer Res Treat 107: 309-330, 2008.

5. Foulkes WD, Reis-Filho JS and Narod SA: Tumor size and survival in breast cancer - a reappraisal. Nat Rev Clin Oncol 7: 348-353, 2010.

6. Anan K, Mitsuyama S, Koga K, Tanabe R, Saimura M, Tanabe Y, Watanabe M, Suehara N, Matsunaga H, Nishihara K, et al: Disparities in the survival improvement of recurrent breast cancer. Breast Cancer 17: 48-55, 2010.
7. Nguyen DX, Bos PD and Massagué J: Metastasis: From dissemination to organ-specific colonization. Nat Rev Cancer 9: 274-284, 2009.

8. Chaffer CL and Weinberg RA: A perspective on cancer cell metastasis. Science 331: 1559-1564, 2011.

9. Morgan BP, Marchbank KJ, Longhi MP, Harris CL and Gallimore AM: Complement: Central to innate immunity and bridging to adaptive responses. Immunol Lett 97: 171-179, 2005.

10. Ricklin D, Hajishengallis G, Yang $K$ and Lambris JD: Complement: A key system for immune surveillance and homeostasis. Nat Immunol 11: 785-797, 2010.

11. Matsumoto M, Takeda J, Inoue N, Hara T, Hatanaka M, Takahashi K, Nagasawa S, Akedo $\mathrm{H}$ and Seya T: A novel protein that participates in nonself discrimination of malignant cells by homologous complement. Nat Med 3: 1266-1270, 1997.

12. Dunkelberger JR and Song WC: Complement and its role in innate and adaptive immune responses. Cell Res 20: 34-50, 2010.

13. Zhou X, Hu W and Qin X: The role of complement in the mechanism of action of rituximab for B-cell lymphoma: Implications for therapy. Oncologist 13: 954-966, 2008.

14. Fishelson Z, Donin N, Zell S, Schultz S and Kirschfink M: Obstacles to cancer immunotherapy: Expression of membrane complement regulatory proteins (mCRPs) in tumors. Mol Immunol 40: 109-123, 2003.

15. Davies A and Lachmann PJ: Membrane defence against complement lysis: The structure and biological properties of CD59. Immunol Res 12: 258-275, 1993.

16. Li B, Lin H, Fan J, Lan J, Zhong Y, Yang Y, Li H and Wang Z: CD59 is overexpressed in human lung cancer and regulates apoptosis of human lung cancer cells. Int J Oncol 43: 850-858, 2013.

17. Hu W, Ge X, You T, Xu T, Zhang J, Wu G, Peng Z, Chorev M, Aktas BH, Halperin JA, et al: Human CD59 inhibitor sensitizes rituximab-resistant lymphoma cells to complement-mediated cytolysis. Cancer Res 71: 2298-2307, 2011.

18. Rus HG, Niculescu FI and Shin ML: Role of the C5b-9 complement complex in cell cycle and apoptosis. Immunol Rev 180: 49-55, 2001.

19. Madjd Z, Pinder SE, Paish C, Ellis IO, Carmichael J and Durrant LG: Loss of CD59 expression in breast tumours correlates with poor survival. J Pathol 200: 633-639, 2003.

20. Li B, Chu X, Gao M and Xu Y: The effects of CD59 gene as a target gene on breast cancer cells. Cell Immunol 272: 61-70, 2011 .

21. Terp MG, Lund RR, Jensen ON, Leth-Larsen R and Ditzel HJ: Identification of markers associated with highly aggressive metastatic phenotypes using quantitative comparative proteomics. Cancer Genomics Proteomics 9: 265-273, 2012.

22. Maio M, Brasoveanu LI, Coral S, Sigalotti L, Lamaj E, Gasparollo A, Visintin A, Altomonte $\mathrm{M}$ and Fonsatti E: Structure, distribution, and functional role of protectin (CD59) in complement-susceptibility and in immunotherapy of human malignancies (Review). Int J Oncol 13: 305-318, 1998.

23. Liu ZB, Hou YF, Min-Dong, Di GH, Wu J, Shen ZZ and Shao ZM: PA-MSHA inhibits proliferation and induces apoptosis through the up-regulation and activation of caspases in the human breast cancer cell lines. J Cell Biochem 108: 195-206, 2009.

24. Du Y, Teng X, Wang N, Zhang X, Chen J, Ding P, Qiao Q, Wang Q, Zhang L, Yang C, et al: NF- $\kappa B$ and enhancer-binding CREB protein scaffolded by CREB-binding protein (CBP)/p300 proteins regulate CD59 protein expression to protect cells from complement attack. J Biol Chem 289: 2711-2724, 2014.

25. Detre S, Saclani Jotti G and Dowsett M: A 'quickscore' method for immunohistochemical semiquantitation: Validation for oestrogen receptor in breast carcinomas. J Clin Pathol 48: 876-878, 1995.

26. Harvey JM, Clark GM, Osborne CK and Allred DC: Estrogen receptor status by immunohistochemistry is superior to the ligand-binding assay for predicting response to adjuvant endocrine therapy in breast cancer. J Clin Oncol 17: 1474-1481, 1999.

27. Du K and Montminy M: CREB is a regulatory target for the protein kinase Akt/PKB. J Biol Chem 273: 32377-32379, 1998.

28. Choi YH, Lee SN, Aoyagi H, Yamasaki Y, Yoo JY, Park B, Shin DM, Yoon HG and Yoon JH: The extracellular signal-regulated kinase mitogen-activated protein kinase/ribosomal S6 protein kinase 1 cascade phosphorylates cAMP response element-binding protein to induce MUC5B gene expression via D-prostanoid receptor signaling. J Biol Chem 286: 34199-34214, 2011. 
29. Boulware MI, Weick JP, Becklund BR, Kuo SP, Groth RD and Mermelstein PG: Estradiol activates group I and II metabotropic glutamate receptor signaling, leading to opposing influences on cAMP response element-binding protein. J Neurosci 25: 5066-5078, 2005.

30. Liu M, Yang YJ, Zheng H, Zhong XR, Wang Y, Wang Z, Wang YG and Wang YP: Membrane-bound complement regulatory proteins are prognostic factors of operable breast cancer treated with adjuvant trastuzumab: A retrospective study. Oncol Rep 32: 2619-2627, 2014.

31. Mamidi S, Cinci M, Hasmann M, Fehring V and Kirschfink M Lipoplex mediated silencing of membrane regulators (CD46, CD55 and CD59) enhances complement-dependent anti-tumor activity of trastuzumab and pertuzumab. Mol Oncol 7: 580-594, 2013.
32. Samatar AA and Poulikakos PI: Targeting RAS-ERK signalling in cancer: Promises and challenges. Nat Rev Drug Discov 13 928-942, 2014

33. Lipp AM, Juhasz K, Paar C, Ogris C, Eckerstorfer P, Thuenauer R, Hesse J, Nimmervoll B, Stockinger H, Schütz GJ, et al: Lck mediates signal transmission from CD59 to the TCR/CD3 pathway in Jurkat T cells. PLoS One 9: e85934, 2014. 